\title{
HIGH TUNING-RATIO MEMS-BASED TUNABLE CAPACITORS FOR RF COMMUNICATIONS APPLICATIONS
}

\author{
J. Jason Yao, SangTae Park, and Jeffrey DeNatale \\ Rockwell Science Center \\ 1049 Camino Dos Rios, Thousand Oaks, CA 91360
}

\begin{abstract}
We have designed, fabricated, and experimentally tested a high tuning-ratio MEMS-based tunable capacitor for RF communications applications. The tunable capacitor is made of single crystal silicon using anisotropic deep silicon etching techniques like the inductively coupled plasma (ICP). The capacitor structure is subsequently released and sputter coated with a metal thin film for improved electrical performances. As application-determined parameters, the nominal base capacitance (unbiased) is designed to be between $1 \mathrm{pf}$ and $5 \mathrm{pf}$, and the tuning ratio has been demonstrated to be in excess of $300 \%$
\end{abstract}

\section{INTRODUCTION}

Tunable capacitors, or varactors, are widely used in RF communications applications for low-noise parametric amplifiers, harmonic frequency generators, and frequency controllers such as voltage controlled oscillators (VCO). Conventional solid-state varactors are made in either silicon or gallium arsenide using either p-n or Schottky-barrier junction structures. Several figures of merit used for tunable capacitors include unbiased base capacitance, tuning ratio, equivalent series resistance or quality factor Q, and associated inductance or electrical self-resonance. The value of unbiased base capacitance depends on the circuit requirements of a specific application and the frequency the tunable capacitor is used for. Typical values are on the order of 1 pf. As for the other figures of merit, solid-state components [1] suffer from a small tuning ratio (typically $30 \%$ ), excessive resistive loss caused by large series resistance and thus a low Q (typically a few 10's), and a low electrical self-resonance due to large parasitics if made on silicon substrates. Drastic measures are often required for the solid-state components to improve on one single parameter, e.g., using light power to increase the tuning ratio [2]

Recent efforts within the MEMS field [3-4] have shown promising results in the realization of a MEMS-based high performance tunable capacitor. These devices were made of parallel metal plates, and the capacitance tuning functionality was achieved by adjusting the spacing between the parallel plates via electrostatic means. Some of the shortcomings of a solid-state varactor, such as large resistive losses, have been overcome with the parallel plate MEMS approach. The tuning range, $16 \%$ reported in [3] and $25 \%$ in [4], with a theoretical limit of $50 \%$, however, requires further improvement for many RF applications.

The objective of the work presented here is to develop and demonstrate a MEMS silicon-based tunable capacitor that has a continuous tuning range of at least $300 \%$, or a 3-to-l tuning ratio. Other parametric requirements of the tunable capacitor include a high $\mathrm{Q}$ factor or a minimum equivalent series resistance, and a high electrical self-resonant frequency or a small inductance value. This paper will discuss in detail the design, modeling, fabrication, and experimental results obtained thus far. The combination of these MEMS performance advantages, along with others including smaller size, lower weight, lower manufacturing cost via batch fabrication techniques, improved linearity, and reduced parts count, should make the MEMS device a suitable and attractive replacement for solid-state varactors in a wide range of $R F$ communications applications.

\section{DEVICE DESIGN}

The design of the MEMS tunable capacitor is tightly oriented around its objective to replace solid-state varactors in an existing military application. Although the concept of a MEMS tunable capacitor can be applied to many military and commercial applications, and consequently the device can be designed quite differently to meet the different needs of various applications, the work described in this paper has a focused set of parametric goals since its application is defined and specific. The theory behind the design is thus strictly application-driven, while the MEMS technology is both enabling and enhancing.

Table 1 lists a set of key device parameters. These parameters define the design-space for the tunable capacitor, which will be further illustrated through the detailed discussions in the following sub-sections.

\begin{tabular}{|l|c|}
\hline \multicolumn{1}{|c|}{ Parameter } & Performance \\
\hline Tuning Ratio & 3 to 1 \\
\hline | Base Capacitance (unbiased) & $1-5 \mathrm{pf}$ \\
\hline Tuning Voltage & $<15 \mathrm{~V}$ \\
\hline Quality Factor Q & $100 @ 400 \mathrm{MHz}$ \\
\hline Electrical Self-Resonance & $5 \mathrm{GHz}$ \\
\hline
\end{tabular}

Table 1: Key parameters for the tunable capacitor define the device's fundamental design-space.

\section{Tuning Range}

The electrostatic actuation scheme is predetermined as the preferred choice of actuation for its ultra low power-consumption characteristic. However, when electrostatic actuation is applied to a cantilevered parallel plate system, the equilibrium between the electrostatic attracting force and the spring back force of the cantilever holds only for a deflection smaller than or equal to onethird of the initial gap between the two parallel plates. Once that limit of one-third of the initial gap is surpassed, the electrostatic force becomes larger than the spring back force, and the cantilever system will snap shut, or become unstable. This limitation imposes a theoretical limit of $50 \%$ on the tuning range of any electrostatically actuated parallel plate system:

$$
\text { MTR }\left(\frac{\varepsilon A}{\left.1 \frac{1}{3}\right) \cdot x_{0} \frac{\varepsilon A}{x_{0}}}=50 \%\right.
$$

where MTR is the maximum tuning range, $\varepsilon$ is the dielectric constant, $\mathrm{A}$ is the surface area, and $\mathrm{x}_{0}$ is the initial gap between the two parallel plates.

Unlike the parallel plate system, the interdigitated "comb" structure does not have the $50 \%$ tuning-range limitation. Its movement relies on the electrostatic force generated by fringing fields at the ends of the "comb" structure, and thus is independent of and not limited by the gap spacing between the two electrodes. This configuration allows a large motion on the order of 10 's $\mu \mathrm{m}$ with a relatively small actuation voltage, thus a large tuning range for the tunable capacitor, and is the preferred choice of design. 


\section{Base Capacitance}

The base capacitance, or the unbiased capacitance, is the minimum capacitance when only a unidirectional drive is used for actuation and tuning. This capacitance needs to be between $1 \mathrm{pf}$ and $5 \mathrm{pf}$, required by the specific application. To achieve this large capacitance, the capacitance density must be maintained as high as possible.

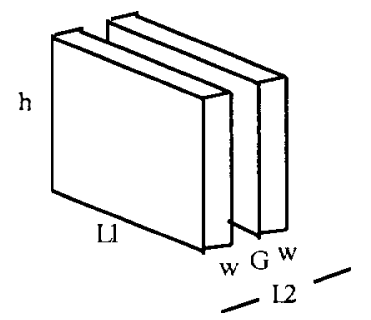

Figure 1: A schematic drawing illustrates a basic parallel plate capacitor structure.

Figure 1 illustrates a unit cell of a basic parallel-plate system, which is a convenient model for general capacitors. The total capacitance, $\mathrm{C}_{0}$, is:

$$
\mathrm{C}_{0}=\left[\varepsilon^{*} \mathrm{~h}^{*} \mathrm{~L}_{1} / \mathrm{G}\right]^{*}\left[\mathrm{~L}_{2} /(\mathrm{G}+\mathrm{w})\right]
$$

or, the capacitance per unit area is:

$$
\mathrm{C}_{0} /\left(\mathrm{L}_{1}{ }^{*} \mathrm{~L}_{2}\right)=\varepsilon^{*} \mathrm{~h} /\left[\mathrm{G}^{*}(\mathrm{G}+\mathrm{w})\right]
$$

Equation 3 demonstrates conclusively that the aspect ratio (height, $\mathrm{h}$, over lateral dimensions, $\mathrm{G}$ or w) must be kept large to maximize the overall capacitance per unit area. This conclusion suggests that the deep anisotropic silicon etch technique using inductively coupled plasma (ICP) is an excellent manufacturing technology for the tunable capacitor device.

\section{Tuning Voltage and Microphonics}

The tradeoff between the tuning voltage and microphonics is a key design issue for the tunable capacitor. With a predetermined capacitance, the required tuning voltage for a certain tuning range depends on the spring constant of the system. To reduce the tuning voltage, the spring constant needs to be kept small. However, an excessively small spring constant will result in unwanted microphonics related problems, and cause the device to be sensitive to environmental vibrations.

In this design work, the focus is on minimizing microphonics while maintaining the tuning voltage within an allowed range predetermined by the system employing the tunable capacitor. Once the tuning voltage is determined, the spring constant can also be determined thereafter. The effort is then focused on minimizing the device mass without compromising the overall capacitance. A mechanical resonant frequency of $20 \mathrm{kHz}$ is determined to be sufficiently high for the tunable capacitor applications. Table 2 illustrates two possible but extreme scenarios where sufficient resolutions can still be maintained for a design where the maximum tuning range of 3 to 1 is achieved with a device movement of $10 \mu \mathrm{m}$. For the specific application the tunable capacitor is designed, a resolution of 9 bit is sufficient.

\begin{tabular}{|l|l|l|}
\hline & Scenario 1 & Scenario 2 \\
\hline Vibration & $10 \mathrm{~g}$ & $1 \mathrm{~g}$ \\
\hline Resonant Freq. & $20 \mathrm{kHz}$ & $20 \mathrm{kHz}$ \\
\hline Deflection & $6 \mathrm{~nm}$ & $0.6 \mathrm{~nm}$ \\
\hline Tuning Range & $10 \mu \mathrm{m}$ & $10 \mu \mathrm{m}$ \\
\hline Resolution & $10 \mathrm{bit}$ & $14 \mathrm{bit}$ \\
\hline
\end{tabular}

Table 2: Microphonics presents minimal effects on the MEMS based tunable capacitor.
Equivalent Series Resistance and Quality Factor $Q$

The equivalent series resistance and quality factor, $\mathrm{Q}$, are two interdependent parameters, used to partially describe the electrical performance of a capacitor. The quality factor is defined as the ratio between the imaginary term of a capacitor and its real term, or:

$$
\mathrm{Q}=(\omega \mathrm{CR})^{-1}
$$

where $\mathrm{R}$ is the equivalent series resistance of a capacitor.

Single crystal silicon is a preferred mechanical material for the tunable capacitor for the reasons mentioned previously, including the high aspect ratio achievable with ICP and its other superior mechanical properties. However, silicon is not a good conductor to provide a low equivalent series resistance and a high Q. Highly doped silicon is especially cautioned because its conductivity, though higher, is still not high enough to be used as a good electrical conductor for the capacitor. In addition, the increased stress level in a heavily doped silicon material may present other challenges in manufacturing of a large MEMS structure. A preferred embodiment is to use silicon as the main mechanical material, and subsequently coat the silicon structure with a layer of metal thin film, such as aluminum, for its good electrical conductivity. Since the metal thin film is a small portion of the metal-silicon structure, the overall device behaves mechanically like single crystal silicon and electrically like aluminum, and thus combines the advantages of the two materials.

\section{Inductance and Electrical Self-Resonance}

The associated inductance within a capacitor and the capacitor's electrical self-resonance are two other interdependent parameters often used to describe the capacitor's RF performance. The electrical self-resonance, $\omega_{0}$, is defined as:

$$
\omega_{0}=(\mathrm{LC})^{-1 / 2}
$$

where $\mathrm{L}$ is the associated inductance within a capacitor, C. Since as the frequency increases, the impedance of a capacitor decreases while that of an associated inductor increases, the overall "capacitor" device becomes inductive when frequency is higher than the self-resonant frequency. Therefore, the self-resonant frequency of a capacitor determines the frequency range in which the capacitor can be used effectively.

To have a high electrical self-resonance, a capacitor must have its associated inductance as small as possible. This involves detailed design to address the tradeoffs between the issues in the mechanical domain and those in the electrical domain, and is beyond the scope of this presentation.

With the confinements of the parametric requirements listed above, the tunable capacitor design is graphically illustrated in Fig. 2. This design presents a two-terminal device solution, which can be schematically illustrated as in Fig. 3(a). The RF signal shares the same two terminals with the biasing or tuning voltage. This configuration resembles very much a solid-state varactor diode, in which biasing choke is needed to isolate the RF signal and the tuning voltage. A more integration-compatible solution that would eliminate the need of large biasing inductors is schematically represented in Fig. 3(b), where a separate tuning capacitor is used. This tuning capacitor can be made much larger than the RF capacitor for linearity purposes, and is mechanically joined with the RF capacitor at the shared ground terminal. Movements generated by the tuning capacitor will thus cause mechanical motion in the RF capacitor, and hence realize the tuning capability. Figure 4 shows a micrograph of such a three-terminal tunable capacitor device. Note that due to the complexity in biasing, solidstate varactors are normally used with one terminal grounded. Hence, the three-terminal configuration does not present any 
additional constraints to its applications as a replacement to the solid-state varactors.

(a)

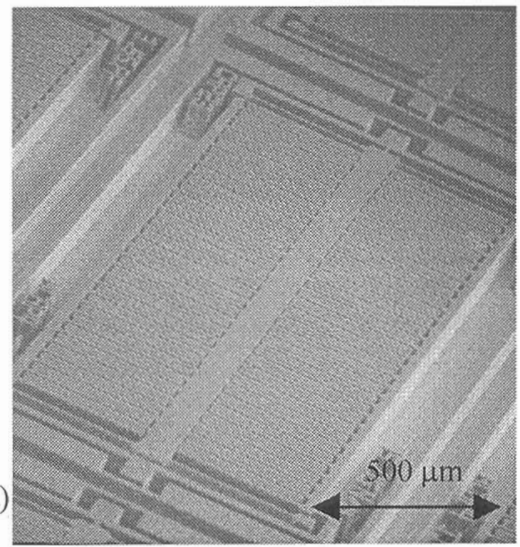

(b)

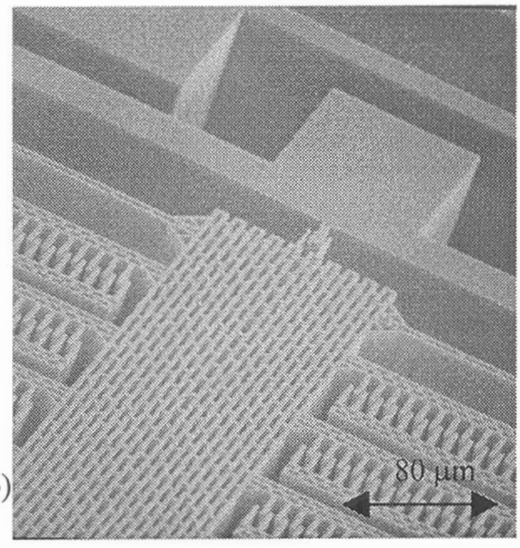

(c)

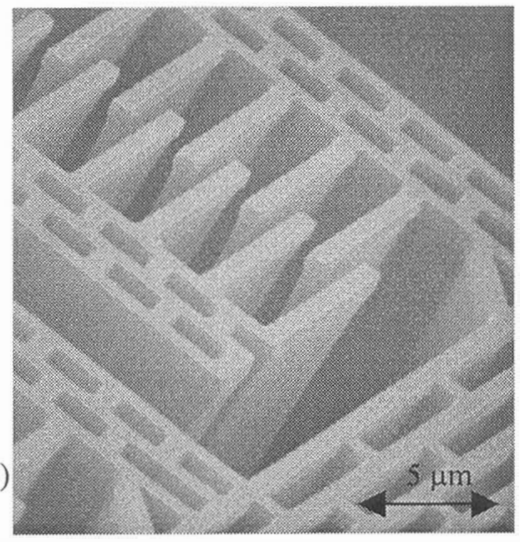

Figure 2: Scanning electron micrographs with three different magnifications showing perspective views of a MEMS-based tunable capacitor. The high aspect-ratio and the anisotropic sidewall profile provided by ICP are critical to the performance of the tunable capacitor.

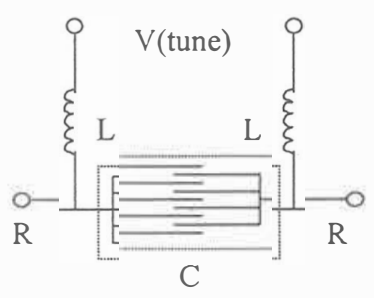

(a)

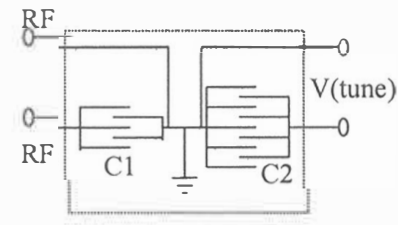

(b)
Figure 3: Schematic illustrations of MEMS tunable capacitors. The dashed boxes highlight the MEMS portion.

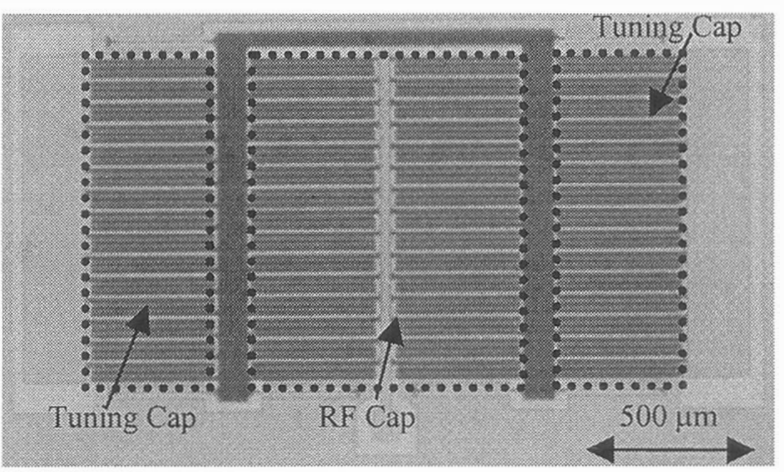

Figure 4: Top view micrograph of a three-terminal tunable capacitor that offers independent tuning and RF connections.

\section{EXPERIMENT AND RESULTS}

The tunable capacitor typically occupies an area of $1 \mathrm{~mm}^{2}$, and is comprised of a large array of interdigitated "comb" or "finger" structures, which are $2-\mu \mathrm{m}$ in width, $2-\mu \mathrm{m}$ spaced, and nominally $30 \mu \mathrm{m}$ in height, Fig. 2. By applying a tuning voltage between the "fingers", an electrostatic force is created to move the suspended structures relative to each other and to change the overlapping distance between the "fingers", and thus the total capacitance. The single crystal silicon device is created using anisotropic deep silicon etching techniques like the inductively coupled plasma (ICP). The starting material is a silicon on insulator (SOI) wafer, with the device silicon layer approximately $30 \mu \mathrm{m}$ thick and a silicon dioxide interlayer of $2 \mu \mathrm{m}$. Figure 5 schematically illustrates the process sequence.

(a)

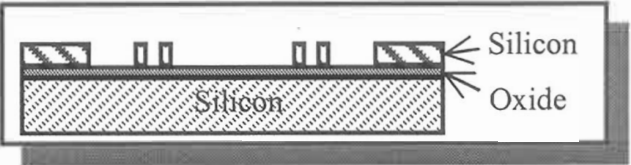

(b)

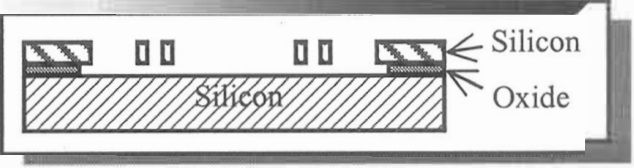

(c)

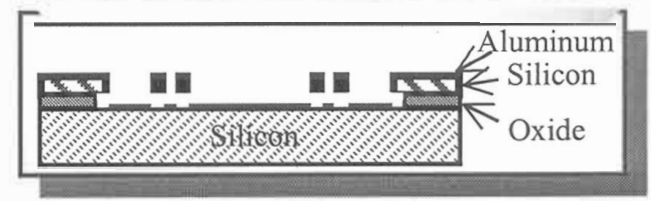

Figure 5: Schematic illustration of the 1-mask process sequence.

The structural pattern of the tunable capacitor design is first transferred to the device silicon layer using photolithography and ICP anisotropic etch, stopping on the underlying silicon dioxide layer, Fig. 5(a). Figure 6 is a scanning electron micrograph of a cross sectional view of the silicon lines and spacing created using such technique. The nominal line width and spacing are $2 \mu \mathrm{m}$, and the silicon height in this particular sample is $20 \mu \mathrm{m}$. The buried silicon dioxide layer is then selectively removed using hydrofluoric acid, and dried in a super critical carbon dioxide vessel, Fig. 5(b). A coat of aluminum is sputtered on after the structural release to reduce the equivalent series resistance of the tunable capacitor device. During the metal sputtering, the suspended structure naturally creates an electrical discontinuity between the electrodes within the capacitor and between the capacitor and the substrate. No further metal lithography or etching steps are needed. This results in a single-mask MEMS process that provides the structure, the isolation and the interconnect metallization. 


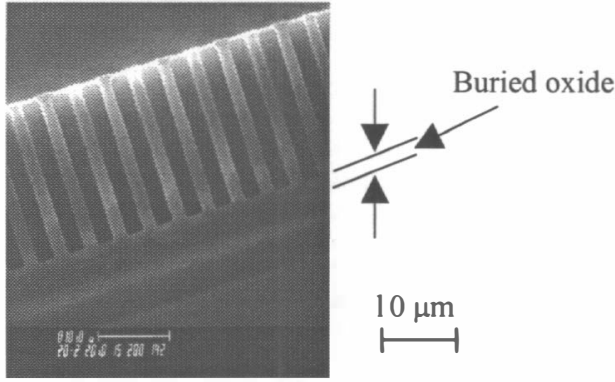

Figure 6: Cross sectional view of silicon structures, 2 um line and spacing, on buried silicon dioxide layer, created using anisotropic deep silicon etch.

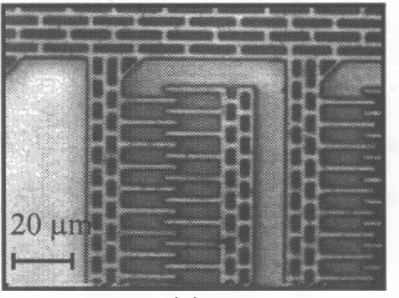

(a)

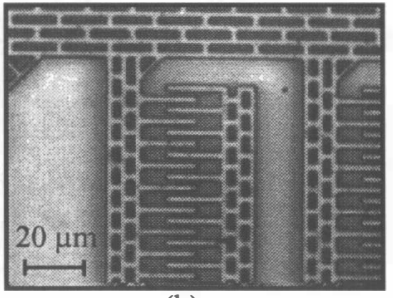

(b)
Figure 7: A portion of the tunable capacitor with (a) $0 \mathrm{~V}$ and (b) $15 \mathrm{~V}$ tuning voltage.

The device has been tested with a DC tuning voltage. Continuous tuning has been observed by varying the tuning voltage, and no resonant operational mode is required. Figure 7 shows a top view of a portion of the tunable capacitor device with the tuning voltage at (a) $0 \mathrm{~V}$ and (b) $15 \mathrm{~V}$. The increase in the overlapping distance between the fingers indicates that a tuning ratio of 3 to 1 has been achieved. Alternatively, the capacitance tuning can be characterized by extracting the imaginary part of the Y-parameter out of the measured S-parameters. Figure 8 is a plot of the $\mathrm{Y}$-parameter as a function of the sweeping signal frequency from $30 \mathrm{MHz}$ to $400 \mathrm{MHz}$. A capacitance change from $3.28 \mathrm{pf}$ with $0 \mathrm{~V}$ tuning voltage to $6.44 \mathrm{pf}$ with $5 \mathrm{~V}$ tuning voltage has been observed - a tuning ratio of $\sim 200 \%$ for this particular test.

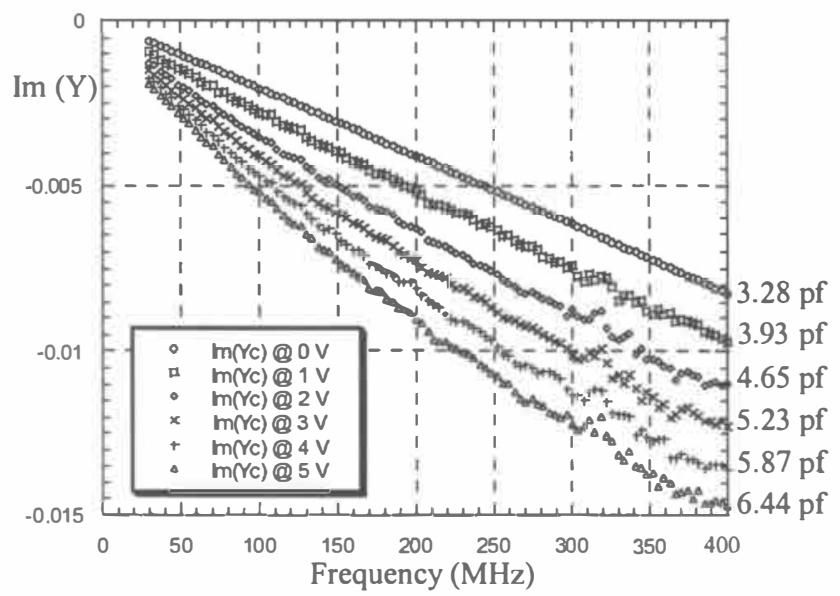

Figure 8: Imaginary part of the extracted Y-parameter from the measured S-parameters as a function of the sweeping signal frequency, showing capacitance tuning of about $200 \%$ with $5 \mathrm{~V}$.

Due to the nature of this 1-mask process, the capacitor structure is only about $2 \mu \mathrm{m}$ away from the underlying silicon substrate. This configuration presents a large parasitic effect in the RF performance of the tunable capacitor. Figure 9(a) illustrates this parasitic effect using S11 in a Smith chart, for a frequency sweep from $30 \mathrm{MHz}$ to $2 \mathrm{GHz}$. The overall shape, along with the small, local resonant loops, is a clear indication of the unwanted parasitic effect. Based on this learning, we have decided to remove the portion of the silicon substrate directly underneath the tunable capacitor with a second masking step to minimize the parasitic effect. Dramatic improvements have been observed as shown in Fig. 9(b), which is a S11 plot for a frequency sweep from $30 \mathrm{MHz}$ to $6 \mathrm{GHz}$. This improved tunable capacitor exhibits a near-ideal capacitive behavior with an electrical self-resonance as high as $5 \mathrm{GHz}$.

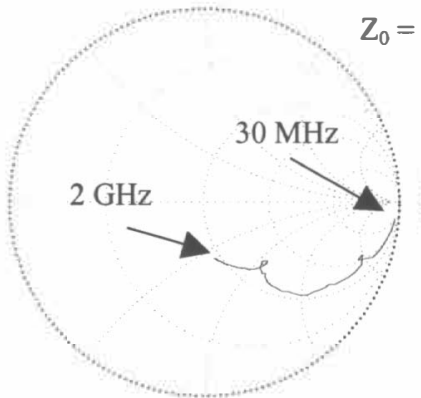

(a) (b)

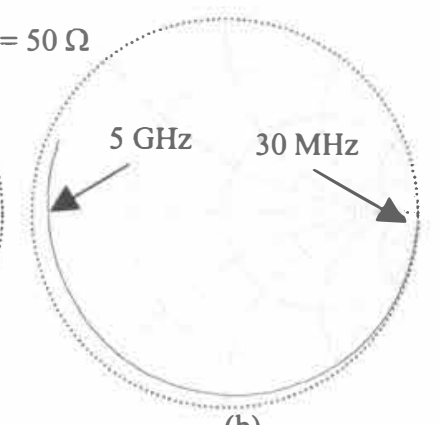

Figure 9: SII plots showing (a) strong parasitic effects with silicon substrate intact, and (b) minimal parasitic effects with portions of the silicon substrate removed.

\section{CONCLUSION}

The MEMS tunable capacitor presented in this paper uses an interdigitated "comb" structure approach, and eliminates the $50 \%$ tuning limit in the parallel plate approach. In addition to the advantages demonstrated in the parallel-plate MEMS approach, we have experimentally demonstrated a large tuning ratio in excess of $300 \%$. Minimal parasitic effects have been achieved by removing portions of the underlying silicon substrate. It should be noted that these tunable capacitors are tuned in a non-vacuum environment with a small tuning voltage less than $15 \mathrm{~V}$ of a frequency from $\mathrm{DC}$ up to the mechanical resonance of the structures, which is typically between $5 \mathrm{kHz}$ and $10 \mathrm{kHz}$. The MEMS tunable capacitor presented here does not use resonant mode of tuning operation, which is not acceptable for most RF applications.

\section{ACKNOWLEDGEMENTS}

This work is supported by DARPA/ETO MEMS Program under BAA 96-19, Contract \# F30602-97-C-0091. The authors also express their thanks to the RSC Clean Room Staff and Test Engineers for their help and support in the development of this project.

\section{REFERENCES}

[1] M. Soyuer, K. A. Jenkins, J. N. Burghartz, and M. D. Hulvey, "A 3V $4 \mathrm{GHz}$ nMOS Voltage-Controlled Oscillator with Integrated Resonator," in Technical Digest - IEEE International Solid-State Circuit Conference, Feb. 1996, pp. 394-395

[2] D-W Kim and Y-S Kwon, "Photoresponse of Area-Variable Varactor Diode," Electronics Letters, v 32, n 5, Feb. 29, 1996, pp. 466-467

[3] D. J. Young and B. E. Boser, "A Micromachined Variable Capacitor for Monolithic Low-Noise VCOs," in Technical Digest - Solid State Sensor and Actuator Workshop, 1996, pp. 86-89

[4] A. Dec and K. Suyama, "Micromachined Varactor with Wide Tuning Range," Electronics Letters, v 33, n 11, May 22, 1997, pp. 922-924 